\title{
Numerical interpretation of TRS-PCR profiling results for Escherichia coli strains isolated from patients with bacteriuria in Lodz region, Poland
}

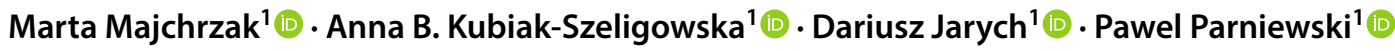

Received: 21 February 2019 / Accepted: 20 June 2019 / Published online: 25 June 2019

(c) The Author(s) 2019

\begin{abstract}
With the multiplicity of existing methods to track $E$. coli infections, it still seems necessary to seek new, better and/or complementary ways for epidemiological investigations. Particularly, fast, cheap, effective and reproducible methods providing easily comparable results are needed. Our previous studies showed that the use of TRS-PCR is an effective molecular tool in $E$. coli epidemiology. In this paper, we have developed a unique classification scheme in which an individual TRS-PCR pattern is assigned a numerical value. This approach allows for rapid interpretation of the results obtained from several similarity dendrograms. Using this approach, based on CAC-PCR, GTG-PCR and CGG-PCR, we obtained 52, 86 and 99 different numerical types for the 124 analyzed uropathogenic $E$. coli strains, respectively. This allowed for the identification of 121 unique isolates differing in at least one TRS-PCR class. In this approach, we got numerical results, easy to sort and interpret, allowing easier analysis of these strains.
\end{abstract}

Keywords Microsatellites $\cdot$ TRS-PCR profiling $\cdot$ E. coli

$\begin{array}{ll}\text { Abbreviations } \\ \text { TRS-PCR } & \text { Trinucleotide repeat sequences-based PCR } \\ \text { CAC } & \text { GTG-, CGG-PCR - CAC, GTG or CGG } \\ & \text { repeats-based PCR, respectively } \\ \text { IPEC } & \text { Intestinal pathogenic E. coli } \\ \text { ExPEC } & \text { Extraintestinal pathogenic } E \text {. coli } \\ \text { UPEC } & \text { Uropathogenic } E \text {. coli } \\ \text { NMEC } & \text { Neonatal meningitis } E \text {. coli } \\ \text { APEC } & \text { Avian pathogenic } E \text {. coli } \\ \text { SEPEC } & \text { Septicemia causing } E . \text { coli } \\ \text { UTI } & \text { Urinary tract infection } \\ \text { VF } & \text { Virulence factors }\end{array}$

Marta Majchrzak and Anna B. Kubiak-Szeligowska have contributed equally to this work.

Electronic supplementary material The online version of this article (https://doi.org/10.1007/s11033-019-04932-2) contains supplementary material, which is available to authorized users.

Pawel Parniewski

pparniewski@gmail.com; pparniewski@cbm.pan.pl

1 Institute of Medical Biology, Polish Academy of Sciences, 106 Lodowa Str, 93-232 Lodz, Poland
MLVA
Multi-locus variable-number tandem repeat analysis
MLST Multi-locus sequence typing
PFGE Pulsed-filed gel electrophoresis

\section{Introduction}

Pathogenic Escherichia coli is an extremely versatile and heterogeneous group of strains which poses a threat to people's health and life from all over the world. Based on the site of induced infection and virulence attributes, this group can be subdivided into intestinal pathogenic E. coli (IPEC) and extraintestinal pathogenic E. coli (ExPEC) strains [1-3]. The most important ExPEC pathotypes, that are associated with human diseases are uropathogenic E. coli (UPEC), neonatal meningitis $E$. coli (NMEC), avian pathogenic $E$. coli (APEC) and septicemia causing E. coli (SEPEC) [1-8].

Majority of urinary tract infections (UTI) are caused by uropathogenic $E$. coli strains, which are highly adapted not only to live in the urinary tract but also to evade the host's defense mechanisms and immune responses [2, 7, 9]. UPEC pathotype constitutes very diverse group of strains with a wide range of virulence factors $[1,2,4,6,10]$. Moreover, not only bacterial factors determine such infections but also 
host factors are involved in this process $[2,7]$. As the number of UPEC strains resistant to antibiotics is increasingly growing and many people are immunocompromised or become sensitive to these pathogens, costs of medical care and other indirect costs escalate every year [7, 10, 11]. It was estimated that every year 130-170 million cases of UTI were registered, in addition to that, over $80 \%$ of them were UPEC induced $[5,11]$.

Molecular epidemiology of $E$. coli infections is based mainly on multi-locus variable-number tandem repeat analysis (MLVA), multi-locus sequence typing (MLST) and pulsed-field gel electrophoresis (PFGE) [8, 12-14]. PFGE is considered as a "gold standard" for epidemiology and strain typing for many bacterial species, including E. coli, but is also time-consuming and results obtained from different laboratories are problematic to compare [13, 14].

In our laboratory, we have developed an effective method for the differentiation and genotyping of bacterial strains called TRS-PCR [15-19]. Trinucleotide repeat sequences are kind of microsatellites dispersed in genome of every organism. This test was successfully used to detect associations between CGG-PCR and GTG-PCR fingerprints and specific E. coli properties such as phylogroups and virulence profiles [15-19]. The method is simple, fast, reproducible and inexpensive. However, a large diversity of fingerprint profiles obtained from various TRS-PCR tests and the need to compare them, in our opinion, required development of a new strategy to facilitate the interpretation of results. Here, we propose a new approach for strain typing of E. coli based on three separate TRS-PCR tests using CAC-PCR, GTGPCR and CGG-PCR profiling. In this technique, each unique TRS-PCR profile is assigned a number. Based on the high reproducibility and also the stability of fingerprints, each strain can be assigned a set of three numbers corresponding to three TRS classes (Table 1). The effectiveness of the proposed approach was tested on a collection of $124 \mathrm{E}$. coli strains isolated from patients with urinary tract infections.

\section{Materials and methods}

\section{Bacterial strains}

One hundred and twenty-four E. coli strains were isolated from patients with urinary tract infections between June 2005 and September 2006 and originated from various wards of Military Teaching Hospital No. 2, Medical University of Lodz, Poland. Information about the date of isolation, ward/department, sex of the patient, the scale of bacteriuria and specified serotype are listed in Table 2 . This collection of strains has been redefined as the spectrum of virulence factors had been extended $[15,16]$, phylogenetic affiliation had been performed according to the new Clermont's protocol [20]. Also, isolates were serotyped according to the manufacturer's protocols for E. coli (O pool and O single antisera, Statens Serum Institut SSI Diagnostica, Denmark) (Fig. 1S.B).

\section{Bacterial growth and genomic DNA isolation}

All E. coli strains were grown overnight in a liquid LB broth at $37^{\circ} \mathrm{C}$ with agitation at $120 \mathrm{RPM}$. For genomic DNA isolation and purification, GenElute Bacterial Genomic DNA Kit was used according to the manufacturer's protocol (Sigma-Aldrich, St. Louis, MO). Each of the genomic DNA sample was measured spectrophotometrically at $260 \mathrm{~nm}$ (BioPhotometer, Eppendorf, Germany) in order to estimate its quantity and quality. The concentration of DNA samples used in the study was $20 \mathrm{ng} / \mu \mathrm{l}$.

\section{Phylogenetic structure}

Phylogenetic analyses were carried out exactly according to the Clermont's E. coli phylo-typing scheme [16]. The whole methodology, such as, sequences of primers, their concentrations and phylo-group detection schemes was performed as described [20] (Fig. 1S.A).

\section{Virulence factors repertoire}

Detection of the virulence factors was performed exactly as it was published elsewhere [16]. The presence of uropathogenic virulence factors was determined using sequences of primers previously published [15]. Detection of intestinal virulence factors typical for different pathovars was performed according to the Müller et al. [21]. The presence of additional five virulence genes-iroN, fyuA, iutA, sat and tsh was determined. Two separate PCRs were performed. The first multiplex PCR reaction was designed to determine the presence of $i r o N$, fyuA and iutA genes. Sequences of primers for iroN detection were published by Bonacorsi et al. [22] and sequences of primers for detection of fyuA and iutA were published by Johnson and Stell [4]. The second multiplex PCR was designed to determine the presence of sat, as described by Restieri et al. [23] and $t s h$, as described by Moulin-Schouleur et al. [24] (Fig. 1S.C).

\section{TRS-PCR profiling and reproducibility analysis}

For each strain, three separate TRS-PCR profiles were generated based on the presence of CAC, GTG, and CGG motifs in used primers. Conditions for GTG-PCR and CGG-PCR reactions, primers sequences, electrophoresis, reproducibility analysis, determination of diversity indices and bioinformatic analyses were performed as described elsewhere [15-19]. Conditions for CAC-PCR were the same as for GTG-PCR 
Table 1 Assignment of three separate TRS-PCR classes for uropathogenic E. coli strains based on CAC-, GTG- and CGG-PCR profiling. Strains sharing identical TRS-PCR profiles and strains derived from the same patients were shown separately

\begin{tabular}{|c|c|c|c|c|c|}
\hline Key & CAC class & GTG class & CGG class & Phylotype & Virulence profile \\
\hline \multicolumn{6}{|c|}{ The differentiated group of strains } \\
\hline $\mathrm{U} 032$ & 1 & 2 & 71 & A & None of the tested \\
\hline U 104 & 2 & 16 & 40 & B1 & papC, fimG/fimH, fyuA, iutA, iroN \\
\hline U 001 & 3 & 1 & 80 & A & None of the tested \\
\hline U 037 & 3 & 51 & 51 & $\mathrm{C}$ & fimG/fimH, astA, fyuA \\
\hline U 038 & 3 & 66 & 5 & $\mathrm{C}$ & fimG/fimH, astA, fyuA \\
\hline U 043 & 4 & 43 & 36 & A & iutA, iroN, tsh \\
\hline U 034 & 4 & 53 & 77 & A & fimG/fimH, astA \\
\hline U 029 & 5 & 36 & 84 & $\mathrm{D}$ & fimG/fimH, astA, fyuA \\
\hline U 091 & 6 & 59 & 24 & A & fyuA \\
\hline U 128 & 8 & 35 & 75 & B1 & fimG/fimH, iutA, iroN \\
\hline U 003 & 9 & 54 & 23 & A & papC \\
\hline U 076 & 10 & 32 & 50 & B1 & fimG/fimH, iroN \\
\hline U 092 & 11 & 58 & 6 & A & None of the tested \\
\hline U 025 & 12 & 40 & 96 & $\mathrm{~F}$ & fimG/fimH, fyuA, iutA, iroN, tsh \\
\hline U 116 & 13 & 40 & 34 & $\mathrm{~F}$ & fimG/fimH, astA, fyuA, iutA, iroN, tsh \\
\hline $\mathrm{U} 023$ & 14 & 38 & 40 & B1 & papC, sfaD/sfaE, cnf1, hly1, fimG/fimH, astA, fyuA, iroN \\
\hline U 052 & 15 & 34 & 78 & B1 & fimG/fimH, astA, iutA, iroN \\
\hline U 004 & 16 & 62 & 91 & A & fimG/fimH \\
\hline U 107 & 17 & 21 & 27 & B1 & hly1, fimG/fimH \\
\hline $\mathrm{U} 068$ & 18 & 15 & 52 & B1 & fimG/fimH \\
\hline U 009 & 19 & 3 & 30 & A & fimG/fimH, astA, iroN \\
\hline U 011 & 19 & 42 & 38 & A & fimG/fimH, fyuA \\
\hline U 044 & 19 & 51 & 45 & $\mathrm{C}$ & fimG/fimH, astA, fyuA, iutA, iroN \\
\hline U 073 & 19 & 57 & 32 & A & fimG/fimH, iroN \\
\hline U 079 & 19 & 57 & 39 & A & papC, sfaD/sfaE, cnf1, usp, fimG/fimH, fyuA, iroN \\
\hline U 041 & 19 & 58 & 75 & A & fimG/fimH \\
\hline U 060 & 19 & 62 & 74 & A & fimG/fimH, fyuA, iutA \\
\hline U 099 & 19 & 67 & 82 & A & fimG/fimH, fyuA, iroN \\
\hline $\mathrm{U} 005$ & 20 & 64 & 32 & A & None of the tested \\
\hline U 106 & 21 & 65 & 72 & A & fimG/fimH \\
\hline U 062 & 22 & 49 & 31 & A & fimG/fimH \\
\hline U 113 & 22 & 61 & 37 & A & fimG/fimH, astA \\
\hline U 071 & 23 & 48 & 86 & A & fimG/fimH, iutA, iroN \\
\hline U 074 & 24 & 18 & 32 & A & fimG/fimH \\
\hline U 059 & 25 & 68 & 43 & $\mathrm{~F}$ & None of the tested \\
\hline U 117 & 25 & 69 & 29 & $\mathrm{~F}$ & fimG/fimH, fyuA, iutA, iroN, tsh \\
\hline U 015 & 26 & 27 & 74 & $\mathrm{~F}$ & fimG/fimH, fyuA, iutA \\
\hline U 007 & 27 & 22 & 83 & $\mathrm{~F}$ & fimG/fimH, iutA \\
\hline U 100 & 27 & 23 & 85 & $\mathrm{~F}$ & fimG/fimH, astA, iutA, iroN \\
\hline U 012 & 27 & 24 & 89 & $\mathrm{~F}$ & papC, fimG/fimH, fyuA, iutA \\
\hline $\mathrm{U} 082$ & 28 & 80 & 64 & B2 & sfaD/sfaE, cnf1, usp, hly1, fimG/fimH, fyuA, iroN \\
\hline U 114 & 28 & 81 & 58 & B2 & papC, sfaD/sfaE, cnf1, usp, hly1, fimG/fimH, fyuA, iutA, iroN \\
\hline U 018 & 29 & 72 & 59 & B2 & sfaD/sfaE, cnf1, usp, hly1, fimG/fimH, fyuA, iutA, iroN \\
\hline U 090 & 30 & 74 & 12 & B2 & papC, sfaD/sfaE, cnf1, usp, hly1, fimG/fimH, astA, fyuA, iroN \\
\hline U 086 & 30 & 83 & 88 & $\mathrm{~B} 2$ & sfaD/sfaE, usp, fimG/fimH, pic, fyuA, iutA, iroN \\
\hline U 127 & 31 & 9 & 95 & B2 & usp, fimG/fimH, fyuA, iutA, iroN \\
\hline U 055 & 31 & 10 & 93 & A & fimG/fimH, fyuA \\
\hline U 130 & 31 & 20 & 26 & B1 & fimG/fimH \\
\hline
\end{tabular}


Table 1 (continued)

\begin{tabular}{|c|c|c|c|c|c|}
\hline Key & CAC class & GTG class & CGG class & Phylotype & Virulence profile \\
\hline U 045 & 31 & 39 & 25 & B1 & fimG/fimH \\
\hline U 119 & 31 & 47 & 51 & $\mathrm{C}$ & papC, hly 1 , fimG/fimH, fyuA, iutA \\
\hline U 028 & 32 & 76 & 4 & B2 & papC, fimG/fimH, fyuA \\
\hline U 108 & 32 & 77 & 18 & B2 & papC, fimG/fimH, fyuA, iutA, iroN \\
\hline U 095 & 32 & 82 & 56 & B2 & papC, fimG/fimH, fyuA, iutA, iroN \\
\hline U 064 & 33 & 19 & 44 & B1 & fimG/fimH, iroN \\
\hline U 072 & 33 & 25 & 62 & $\mathrm{~B} 2$ & papC, sfaD/sfaE, cnf1, usp, hly1, fimG/fimH, fyuA, iutA, iroN, sat \\
\hline $\mathrm{U} 026$ & 33 & 71 & 67 & B2 & papC, sfaD/sfaE, cnf1, usp, hly1, fimG/fimH, fyuA, iutA, iroN, sat \\
\hline U 087 & 33 & 72 & 6 & B2 & papC, sfaD/sfaE, cnf1, usp, hly1, fimG/fimH, fyuA, iutA, iroN, sat \\
\hline U 089 & 33 & 72 & 10 & $\mathrm{~B} 2$ & sfaD/sfaE, usp, fimG/fimH, fyuA, iroN \\
\hline U 013 & 33 & 72 & 60 & $\mathrm{~B} 2$ & papC, sfaD/sfaE, cnf1, usp, hly1, fimG/fimH, fyuA, iutA, iroN, sat \\
\hline U 046 & 33 & 72 & 61 & $\mathrm{~B} 2$ & papC, sfaD/sfaE, cnf1, usp, hly1, fimG/fimH, fyuA, iutA, iroN, sat \\
\hline U 033 & 33 & 73 & 62 & B2 & papC, sfaD/sfaE, cnf1, usp, hly1, fimG/fimH, fyuA, iroN \\
\hline U 054 & 33 & 74 & 63 & $\mathrm{~B} 2$ & sfaD/sfaE, cnf1, usp, hly1, fimG/fimH, fyuA, iroN \\
\hline U 006 & 33 & 74 & 97 & B2 & sfaD/sfaE, cnf1, usp, hly1, fimG/fimH, fyuA, iroN \\
\hline U 049 & 33 & 85 & 68 & B2 & sfaD/sfaE, cnf1, usp, hly1, fimG/fimH, fyuA, iutA, iroN \\
\hline U 047 & 33 & 85 & 69 & B2 & sfaD/sfaE, cnf1, usp, hly1, fimG/fimH, fyuA, iroN \\
\hline U 063 & 33 & 86 & 1 & B2 & papC, cnf1, usp, hly1, fimG/fimH, fyuA \\
\hline U 105 & 34 & 74 & 97 & $\mathrm{~B} 2$ & papC, sfaD/sfaE, cnf1, usp, hly1, fimG/fimH, fyuA, iroN \\
\hline U 050 & 35 & 9 & 94 & $\mathrm{~B} 2$ & usp, fimG/fimH, fyuA, iutA, iroN, tsh \\
\hline U 080 & 35 & 33 & 35 & B1 & fimG/fimH, iroN \\
\hline U 057 & 36 & 13 & 48 & $\mathrm{C}$ & fimG/fimH, fyuA, iutA \\
\hline U 019 & 36 & 17 & 73 & A & None of the tested \\
\hline $\mathrm{U} 027$ & 36 & 29 & 8 & B2 & papC, sfaD/sfaE, cnf1, usp, fimG/fimH, fyuA, iutA, iroN \\
\hline U 124 & 36 & 37 & 16 & $\mathrm{~B} 2$ & fimG/fimH, fyuA, iutA, sat \\
\hline U 010 & 36 & 41 & 66 & $\mathrm{E}$ & papC, fimG/fimH \\
\hline U 121 & 36 & 52 & 48 & $\mathrm{C}$ & fimG/fimH, iutA, iroN \\
\hline U 083 & 36 & 63 & 15 & B2 & papC, cnf1, hly1, fimG/fimH, fyuA, iutA, iroN \\
\hline U 088 & 36 & 72 & 7 & $\mathrm{~B} 2$ & sfaD/sfaE, cnf1, usp, hly1, fimG/fimH, fyuA \\
\hline U 016 & 36 & 72 & 59 & $\mathrm{~B} 2$ & papC, sfaD/sfaE, usp, fimG/fimH, fyuA, iutA, iroN, sat \\
\hline U 014 & 36 & 74 & 98 & $\mathrm{~B} 2$ & sfaD/sfaE, cnf1, hly1, fimG/fimH, fyuA, iroN \\
\hline U 030 & 36 & 75 & 3 & $\mathrm{~B} 2$ & sfaD/sfaE, cnf1, hly1, fimG/fimH, fyuA, iroN \\
\hline U 085 & 36 & 75 & 11 & $\mathrm{~B} 2$ & papC, sfaD/sfaE, cnf1, usp, hly1, fimG/fimH, astA, fyuA, iroN \\
\hline U 125 & 36 & 75 & 12 & $\mathrm{~B} 2$ & papC, sfaD/sfaE, cnf1, usp, hly1, fimG/fimH, astA, fyuA, iroN \\
\hline U 098 & 36 & 75 & 66 & $\mathrm{~B} 2$ & papC, sfaD/sfaE, cnf1, usp, hly1, fimG/fimH, astA, fyuA, iroN \\
\hline U 093 & 36 & 76 & 4 & $\mathrm{~B} 2$ & papC, fimG/fimH, fyuA, iutA, iroN \\
\hline $\mathrm{U} 021$ & 36 & 77 & 17 & B2 & papC, fimG/fimH, fyuA, iutA, iroN \\
\hline U 040 & 36 & 78 & 2 & $\mathrm{~B} 2$ & papC, sfaD/sfaE, usp, hly1, fimG/fimH, fyuA, iutA, iroN, sat \\
\hline U 035 & 36 & 80 & 59 & B2 & papC, sfaD/sfaE, cnf1, usp, hly1, fimG/fimH, fyuA, iroN \\
\hline U 036 & 36 & 82 & 55 & $\mathrm{~B} 2$ & papC, sfaD/sfaE, cnf1, usp, hly1, fimG/fimH, fyuA, iutA, iroN \\
\hline U 067 & 36 & 84 & 13 & $\mathrm{~B} 2$ & papC, sfaD/sfaE, cnf1, usp, hly1, fimG/fimH, fyuA, iroN \\
\hline U 110 & 37 & 11 & 14 & $\mathrm{~B} 2$ & papC, sfaD/sfaE, cnf1, usp, hly1, fimG/fimH, pic, fyuA, iroN \\
\hline U 111 & 37 & 75 & 1 & $\mathrm{~B} 2$ & papC, sfaD/sfaE, cnf1, usp, hly1, fimG/fimH, fyuA, iutA, iroN, sat \\
\hline U 084 & 37 & 75 & 9 & $\mathrm{~B} 2$ & papC, sfaD/sfaE, cnf1, usp, hly1, fimG/fimH, fyuA, iutA, iroN, sat \\
\hline U 102 & 37 & 78 & 1 & $\mathrm{~B} 2$ & papC, sfaD/sfaE, usp, hly1, fimG/fimH, fyuA, iutA, iroN, sat \\
\hline U 101 & 37 & 84 & 57 & $\mathrm{~B} 2$ & papC, sfaD/sfaE, cnf1, usp, hly1, fimG/fimH, fyuA, iutA, iroN, sat \\
\hline $\mathrm{U} 048$ & 38 & 12 & 54 & $\mathrm{C}$ & fimG/fimH, astA, fyuA, iutA, iroN \\
\hline $\mathrm{U} 024$ & 38 & 28 & 49 & $\mathrm{C}$ & fimG/fimH \\
\hline U 077 & 39 & 17 & 42 & A & astA, iutA, iroN \\
\hline U 039 & 40 & 26 & 1 & $\mathrm{~B} 2$ & papC, sfaD/sfaE, fimG/fimH, fyuA, iutA, iroN \\
\hline
\end{tabular}


Table 1 (continued)

\begin{tabular}{|c|c|c|c|c|c|}
\hline Key & CAC class & GTG class & CGG class & Phylotype & Virulence profile \\
\hline U 097 & 40 & 26 & 57 & B2 & papC, sfaD/sfaE, fimG/fimH, fyuA, iutA, iroN \\
\hline U 066 & 40 & 79 & 1 & $\mathrm{~B} 2$ & papC, sfaD/sfaE, cnf1, hly1, fimG/fimH, fyuA, iroN \\
\hline U 115 & 41 & 46 & 46 & $\mathrm{C}$ & fimG/fimH, iroN \\
\hline U 002 & 42 & 4 & 33 & B1 & fimG/fimH \\
\hline U 109 & 42 & 6 & 87 & B1 & fimG/fimH \\
\hline U 042 & 42 & 44 & 53 & B1 & fimG/fimH, fyuA, iutA, iroN \\
\hline U 120 & 42 & 45 & 79 & B1 & fimG/fimH, iutA, iroN \\
\hline U 031 & 42 & 50 & 28 & B1 & fimG/fimH, iutA, iroN \\
\hline U 096 & 42 & 56 & 76 & B1 & fimG/fimH, iroN \\
\hline U 017 & 43 & 70 & 92 & A & fimG/fimH \\
\hline U 094 & 44 & 7 & 41 & B1 & fimG/fimH, iutA, iroN, tsh \\
\hline U 051 & 45 & 34 & 47 & B1 & fimG/fimH, iutA, iroN \\
\hline U 126 & 46 & 8 & 99 & A & astA, fyuA, iroN \\
\hline U 103 & 47 & 30 & 20 & $\mathrm{D}$ & papC, fimG/fimH, iutA, sat \\
\hline U 061 & 47 & 31 & 21 & $\mathrm{D}$ & fimG/fimH, iutA, iroN \\
\hline U 053 & 48 & 60 & 81 & $\mathrm{D}$ & fimG/fimH, astA, iutA, iroN \\
\hline U 070 & 49 & 30 & 19 & $\mathrm{D}$ & fimG/fimH \\
\hline U 118 & 50 & 30 & 22 & $\mathrm{D}$ & fimG/fimH, iutA, iroN \\
\hline $\mathrm{U} 008$ & 51 & 5 & 70 & $\mathrm{D}$ & fimG/fimH, fyuA \\
\hline U 081 & 52 & 14 & 90 & $\mathrm{D}$ & fimG/fimH, pic, aggR, fyuA, iutA \\
\hline \multicolumn{6}{|c|}{ Pairs of strains identical in TRS-PCR analysis } \\
\hline $\mathrm{U} 020$ & 7 & 55 & 32 & A & astA, iutA, iroN \\
\hline $\mathrm{U} 022$ & 7 & 55 & 32 & A & fyuA, iutA \\
\hline $\mathrm{U} 058$ & 33 & 71 & 62 & B2 & sfaD/sfaE, cnf1, usp, hly1, fimG/fimH, fyuA, iroN \\
\hline U 112 & 33 & 71 & 62 & B2 & papC, sfaD/sfaE, cnf1, usp, hly1, fimG/fimH, fyuA, iroN \\
\hline U 069 & 33 & 72 & 65 & $\mathrm{~B} 2$ & papC, sfaD/sfaE, cnf1, usp, hly1, fimG/fimH, fyuA, iutA, iroN, sat \\
\hline U 075 & 33 & 72 & 65 & $\mathrm{~B} 2$ & papC, sfaD/sfaE, cnf1, usp, hly1, fimG/fimH, fyuA, iutA, iroN, sat \\
\hline \multicolumn{6}{|c|}{ Strains derived from the same patients } \\
\hline $\mathrm{U} 004$ & 16 & 62 & 91 & A & fimG/fimH \\
\hline U 020 & 7 & 55 & 32 & A & astA, iutA, iroN \\
\hline $\mathrm{U} 006$ & 33 & 74 & 97 & $\mathrm{~B} 2$ & sfaD/sfaE, cnf1, usp, hly1, fimG/fimH, fyuA, iroN \\
\hline U 021 & 36 & 77 & 17 & B2 & papC, fimG/fimH, fyuA, iutA, iroN \\
\hline U 011 & 19 & 42 & 38 & A & fimG/fimH, fyuA \\
\hline U 031 & 42 & 50 & 28 & B1 & fimG/fimH, iutA, iroN \\
\hline U 013 & 33 & 72 & 60 & B2 & papC, sfaD/sfaE, cnf1, usp, hly1, fimG/fimH, fyuA, iutA, iroN, sat \\
\hline U 046 & 33 & 72 & 61 & $\mathrm{~B} 2$ & papC, sfaD/sfaE, cnf1, usp, hly1, fimG/fimH, fyuA, iutA, iroN, sat \\
\hline U 014 & 36 & 74 & 98 & B2 & sfaD/sfaE, cnf1, hly1, fimG/fimH, fyuA, iroN \\
\hline U 054 & 33 & 74 & 63 & B2 & sfaD/sfaE, cnf1, usp, hly1, fimG/fimH, fyuA, iroN \\
\hline $\mathrm{U} 032$ & 1 & 2 & 71 & A & None of the tested \\
\hline U 039 & 40 & 26 & 1 & B2 & papC, sfaD/sfaE, fimG/fimH, fyuA, iutA, iroN \\
\hline U 097 & 40 & 26 & 57 & B2 & papC, sfaD/sfaE, fimG/fimH, fyuA, iutA, iroN \\
\hline U 037 & 3 & 51 & 51 & $\mathrm{C}$ & fimG/fimH, astA, fyuA \\
\hline U 050 & 35 & 9 & 94 & B2 & usp, fimG/fimH, fyuA, iutA, iroN, tsh \\
\hline $\mathrm{U} 042$ & 42 & 44 & 53 & B1 & fimG/fimH, fyuA, iutA, iroN \\
\hline U 124 & 36 & 37 & 16 & B2 & fimG/fimH, fyuA, iutA, sat \\
\hline U 055 & 31 & 10 & 93 & A & fimG/fimH, fyuA \\
\hline U 109 & 42 & 6 & 87 & B1 & fimG/fimH \\
\hline U 121 & 36 & 52 & 48 & $\mathrm{C}$ & fimG/fimH, iutA, iroN \\
\hline $\mathrm{U} 062$ & 22 & 49 & 31 & A & fimG/fimH \\
\hline U 096 & 42 & 56 & 76 & B1 & fimG/fimH, iroN \\
\hline
\end{tabular}


Table 1 (continued)

\begin{tabular}{llllll}
\hline Key & CAC class & GTG class & CGG class & Phylotype & Virulence profile \\
\hline U 113 & 22 & 61 & 37 & A & fimG/fimH, astA \\
U 116 & 13 & 40 & 34 & F & fimG/fimH, astA, fyuA, iutA, iroN, tsh \\
\hline
\end{tabular}

except for the primer sequence. Reproducibility of each clustering analyses was calculated by a comparison of the densitometric curve, characteristic of three separate band patterns obtained for the three chosen strains (Pearson coefficient, UPGMA). Amplifications were repeated with the same strain on a different day. The average similarity (SA) of the band pattern obtained for these strains and the standard deviation (SD) values were estimated. The reproducibility rate of the band pattern $(\mathrm{R})$ for each primer was calculated as follows: $\mathrm{R}=\mathrm{SA}-\mathrm{SD}[\%]$ (Table 1S). The PCR amplifications were accomplished using a T-3000 termocycler (Biometra, Goettingen, Germany). The PCR products were separated by horizontal electrophoresis on $1.6 \%$ agarose gel in a $1 \times \mathrm{TAE}$ buffer at $6 \mathrm{~V} / \mathrm{cm}$ operating within $2 \mathrm{~h}$, stained with ethidium bromide and photographed. Subsequently, gels were optimized according to the recommendations provided by BioNumerics 5.0 software (Applied Maths, Belgium) and normalized with regard to a 100 bp Plus DNA size marker (Fermentas, Thermo Scientific Waltham, MA, USA). The three separate fingerprint similarity analyses based on CAC-PCR, GTG-PCR and CGG-PCR profiles using Pearson correlation (optimization $1 \%$, position tolerance $1 \%$ ) and grouping according to the UPGMA algorithm were performed. The reproducibility analysis of CAC-PCR was performed as previously described for CGG and GTG [15-19] and obtained by comparing the three separate fingerprints from three different PCR reactions for the three chosen UPEC strains.

\section{Stability analyses of CAC-, GTG-, CGG-PCR profiles}

Two strains were selected for this study: U 036 isolated from urine and representative of another collection, K 157+isolated from stool. Stability analysis for each of the used primers with the TRS motif was obtained by comparing TRS-PCR fingerprints performed for several passages (initial, fifth, twentyfifth and thirty-ninth) from a series of consecutive subcultures of these strains. The fingerprint similarity analyses based on CAC-PCR, GTG-PCR and CGG-PCR profiles (Fig. 2S) were performed exactly as for TRS-PCR profiling described above and published elsewhere [15-19].

\section{Results}

In previous analyses of E. coli strains, we analyzed CGGand GTG-PCR profiles [16]. In this approach, for the first time we further performed CAC-PCR profiling on genomic DNAs obtained from a collection of 124 E. coli strains. The fingerprint similarity analysis for this assay was performed. The analysis of reproducibility was also performed and it exhibited 95.0\%. Fingerprint patterns with similarity above this value, were treated as identical. Based on analyses using BioNumerics software, each unique profile was assigned a class number (Fig. 1). Next, the GTG-PCR and CGG-PCR tests were conducted on the examined strains. The fingerprint similarity analysis and assignment of class numbers were done in an analogous way. Thereby, 52 CAC-PCR classes, 86 GTG-PCR classes and 99 CGG-PCR classes were obtained. This allowed the separation of 121 unique isolates differing in at least one TRS class (Table 1). The TRS pattern stability studies conducted on multi-generational re-cultivations have shown that the profiles were almost identical, starting from the first re-cultivation to the last one (Fig. 2S). This allowed us to conclude that assigning numbers of classes in such a way was reliable.

In parallel, these results were also confronted with the belonging of strains to phylogenetic groups by applying the Clermont's E. coli phylo-typing improved method scheme [4] and with their repertoire of pathogenic factors $[15,16,20-24]$. The results are shown in the Table 1.

\section{Discussion}

Until now, we have focused more closely on grouping strains in dendrogram clusters due to the presence of the examined traits (VFs, phylogroups). Currently, our new approach focuses on the maximum distinction of the strains in the collection. The proposed assay for $E$. 
Table 2 Epidemiological information about $E$. coli strains used in the study

\begin{tabular}{|c|c|c|c|c|c|}
\hline Key & Department & Date [D-M-Y] & Bacteriuria & Sex & Serotype/O-group \\
\hline U 001 & A & 05.07 .2005 & $10^{5}$ & $\mathrm{~F}$ & NT \\
\hline U 002 & B & 02.07 .2005 & $10^{6}$ & M & NT \\
\hline U 003 & B & 30.06 .2005 & $10^{6}$ & $\mathrm{~F}$ & O-rough \\
\hline U 004 & $\mathrm{D}$ & 29.06 .2005 & $10^{6}$ & $\mathrm{~F}$ & $\mathrm{O} 7$ \\
\hline U 005 & B & 20.06 .2005 & $10^{6}$ & $\mathrm{~F}$ & HH-pool \\
\hline U 006 & $\mathrm{~B}$ & 25.07 .2005 & $10^{6}$ & $\mathrm{~F}$ & NT \\
\hline U 007 & B & 01.08 .2005 & $10^{6}$ & M & $\mathrm{O} 1$ \\
\hline U 008 & D & 19.08 .2005 & $10^{6}$ & $\mathrm{~F}$ & NT \\
\hline U 009 & B & 17.08.2005 & $10^{6}$ & $\mathrm{~F}$ & NT \\
\hline U 010 & $\mathrm{D}$ & 29.08 .2005 & $10^{6}$ & M & NT \\
\hline U 011 & B & 17.08.2005 & $10^{5}$ & $\mathrm{~F}$ & O-rough \\
\hline U 012 & $\mathrm{~B}$ & 30.08 .2005 & $10^{6}$ & M & SS-pool \\
\hline U 013 & $\mathrm{C}$ & 12.09 .2005 & $10^{6}$ & M & $\mathrm{O} 2$ \\
\hline U 014 & $\mathrm{C}$ & 28.09 .2005 & $10^{6}$ & $\mathrm{~F}$ & NT \\
\hline U 015 & $\mathrm{E}$ & 27.09.2005 & $10^{5}$ & $\mathrm{~F}$ & $\mathrm{O} 1$ \\
\hline U 016 & $\mathrm{E}$ & 25.10 .2005 & $10^{3}$ & $\mathrm{M} / \mathrm{CH}$ & O6 \\
\hline U 017 & $\mathrm{~F}$ & 22.10 .2005 & $10^{6}$ & $\mathrm{~F}$ & HH-pool \\
\hline U 018 & B & 20.10 .2005 & $10^{6}$ & $\mathrm{~F}$ & $\mathrm{O} 22$ \\
\hline U 019 & G & 20.10 .2005 & $10^{6}$ & $\mathrm{~F}$ & O9 \\
\hline U 020 & D & 10.10 .2005 & $10^{6}$ & $\mathrm{~F}$ & NT \\
\hline U 021 & B & 10.10 .2005 & $10^{6}$ & $\mathrm{~F}$ & $\mathrm{O} 2$ \\
\hline U 022 & B & 10.10 .2005 & $10^{6}$ & M & NT \\
\hline U 023 & I & $19.10,2005$ & $10^{6}$ & $\mathrm{~F}$ & NT \\
\hline U 024 & B & 18.10 .2005 & $10^{6}$ & M & O8 \\
\hline U 025 & B & 17.10.2005 & $10^{6}$ & $\mathrm{~F}$ & O-rough \\
\hline U 026 & B & 17.10.2005 & $10^{6}$ & $\mathrm{~F}$ & O-rough \\
\hline U 027 & $\mathrm{E}$ & 13.10 .2005 & $10^{5}$ & $\mathrm{~F}$ & O-rough \\
\hline U 028 & B & 14.10 .2005 & $10^{6}$ & $\mathrm{~F}$ & $\mathrm{O} 2$ \\
\hline U 029 & $\mathrm{C}$ & 12.10 .2005 & $10^{5}$ & $\mathrm{~F}$ & O7 \\
\hline U 030 & B & 26.10 .2005 & $10^{6}$ & $\mathrm{~F}$ & NT \\
\hline U 031 & B & 27.10 .2005 & ND & $\mathrm{F}$ & O-rough \\
\hline U 032 & B & 27.10.2005 & $10^{5}$ & $\mathrm{M} / \mathrm{CH}$ & NT \\
\hline U 033 & $\mathrm{D}$ & 28.10 .2005 & $10^{5}$ & $\mathrm{~F}$ & O6 \\
\hline U 034 & B & 30.10 .2005 & $10^{6}$ & $\mathrm{~F}$ & O7 \\
\hline U 035 & $\mathrm{H}$ & 04.11 .2005 & $10^{5}$ & $\mathrm{~F}$ & O6 \\
\hline U 036 & $\mathrm{H}$ & 04.11 .2005 & $10^{5}$ & $\mathrm{~F}$ & O6 \\
\hline U 037 & I & 07.11 .2005 & $10^{5}$ & $\mathrm{~F}$ & NT \\
\hline U 038 & $\mathrm{E}$ & 10.11 .2005 & $10^{6}$ & M & NT \\
\hline U 039 & $\mathrm{E}$ & 10.11 .2005 & $10^{3}$ & $\mathrm{M} / \mathrm{CH}$ & O6 \\
\hline U 040 & $\mathrm{~F}$ & 11.11 .2005 & $10^{3}$ & $\mathrm{~F}$ & O-rough \\
\hline U 041 & G & 14.11 .2005 & $10^{6}$ & $\mathrm{~F}$ & NT \\
\hline U 042 & C & 14.11 .2005 & $10^{5}$ & $\mathrm{~F}$ & O6 \\
\hline U 043 & $\mathrm{H}$ & 05.12 .2005 & $10^{6}$ & $\mathrm{~F}$ & O15 \\
\hline U 044 & B & 06.12 .2005 & $10^{5}$ & M & NT \\
\hline U 045 & $\mathrm{E}$ & 07.12 .2005 & $10^{6}$ & $\mathrm{~F}$ & O-rough \\
\hline U 046 & $\mathrm{E}$ & 02.12 .2005 & $10^{6}$ & M & $\mathrm{O} 2$ \\
\hline U 047 & $\mathrm{E}$ & 30.11 .2005 & $10^{6}$ & $\mathrm{~F}$ & $\mathrm{O} 22$ \\
\hline U 048 & B & 26.11 .2005 & $10^{6}$ & M & NT \\
\hline U 049 & $\mathrm{C}$ & 21.11 .2005 & $10^{6}$ & M & O-rough \\
\hline $\mathrm{U} 050$ & I & 08.01 .2006 & $10^{5}$ & $\mathrm{~F}$ & OO-pool \\
\hline U 051 & $\mathrm{E}$ & 23.11 .2005 & $10^{6}$ & $\mathrm{~F}$ & O-rough \\
\hline
\end{tabular}


Table 2 (continued)

\begin{tabular}{|c|c|c|c|c|c|}
\hline Key & Department & Date [D-M-Y] & Bacteriuria & Sex & Serotype/O-group \\
\hline U 052 & $\mathrm{~J}$ & 18.11.2005 & $10^{6}$ & $\mathrm{~F}$ & PP-pool \\
\hline U 053 & B & 07.01 .2006 & $10^{6}$ & $\mathrm{~F}$ & NT \\
\hline U 054 & $\mathrm{C}$ & 05.01 .2006 & $10^{6}$ & $\mathrm{~F}$ & NT \\
\hline U 055 & $\mathrm{C}$ & 04.01 .2006 & $10^{5}$ & $\mathrm{~F}$ & FF-pool \\
\hline U 057 & $\mathrm{C}$ & 04.01 .2006 & $10^{6}$ & $\mathrm{~F}$ & NT \\
\hline U 058 & $\mathrm{C}$ & 04.01 .2006 & $10^{4}$ & M & $\mathrm{O} 22$ \\
\hline U 059 & $\mathrm{~B}$ & 27.12.2005 & $10^{6}$ & M & NT \\
\hline U 060 & $\mathrm{~N}$ & 20.12 .2005 & $10^{6}$ & $\mathrm{~F}$ & NT \\
\hline U 061 & $\mathrm{E}$ & 21.12 .2005 & $10^{6}$ & $\mathrm{~F}$ & $\mathrm{O} 17$ \\
\hline U 062 & $\mathrm{E}$ & 31.01 .2006 & $10^{6}$ & $\mathrm{~F}$ & WW-pool \\
\hline U 063 & $\mathrm{D}$ & 14.02 .2006 & $10^{6}$ & M & $\mathrm{O} 4$ \\
\hline U 064 & $\mathrm{E}$ & 08.03 .2006 & $10^{6}$ & $\mathrm{~F}$ & NT \\
\hline U 066 & $\mathrm{C}$ & 31.01 .2006 & $10^{6}$ & F & O6 \\
\hline U 067 & $\mathrm{E}$ & 03.02 .2006 & $10^{5}$ & $\mathrm{~F}$ & $\mathrm{O} 75$ \\
\hline U 068 & $\mathrm{E}$ & 13.01.2006 & $10^{6}$ & $\mathrm{~F}$ & O8 \\
\hline U 069 & $\mathrm{G}$ & 06.09 .2006 & $10^{6}$ & F & $\mathrm{O} 2$ \\
\hline U 070 & $\mathrm{E}$ & 16.01 .2006 & $10^{6}$ & F & NT \\
\hline U 071 & $\mathrm{D}$ & 06.09.2006 & $10^{5}$ & F & O-rough \\
\hline U 072 & B & 23.01 .2006 & $10^{5}$ & F & O6 \\
\hline U 073 & $\mathrm{H}$ & 31.01 .2006 & $10^{6}$ & $\mathrm{~F}$ & NT \\
\hline U 074 & $\mathrm{C}$ & 28.01 .2006 & $10^{6}$ & F & NT \\
\hline U 075 & $\mathrm{C}$ & 08.03.2006 & $10^{6}$ & F & $\mathrm{O} 2$ \\
\hline U 076 & $\mathrm{~B}$ & 10.02 .2006 & $10^{5}$ & M & UU-pool \\
\hline U 077 & $\mathrm{~F}$ & 07.03.2006 & $10^{6}$ & $\mathrm{~F}$ & NT \\
\hline U 079 & $\mathrm{D}$ & 15.02 .2006 & $10^{6}$ & M & O-rough \\
\hline U 080 & $\mathrm{~B}$ & 21.02.2006 & $10^{6}$ & M & $\mathrm{O} 8$ \\
\hline U 081 & $\mathrm{D}$ & 22.02 .2006 & $10^{6}$ & M & NT \\
\hline U 082 & $\mathrm{E}$ & 24.02 .2006 & $10^{6}$ & $\mathrm{~F}$ & NT \\
\hline U 083 & $\mathrm{C}$ & 27.02.2006 & $10^{6}$ & F & $\mathrm{O} 25$ \\
\hline U 084 & $\mathrm{C}$ & 06.03.2006 & $10^{6}$ & F & O75 \\
\hline U 085 & $\mathrm{D}$ & 13.03.2006 & $10^{5}$ & F & O6 \\
\hline U 086 & B & 14.03.2006 & $10^{5}$ & M & $\mathrm{O} 2$ \\
\hline U 087 & B & 14.03 .2006 & $10^{6}$ & M & $\mathrm{O} 2$ \\
\hline U 088 & $\mathrm{D}$ & 22.03.2006 & $10^{6}$ & M & O6 \\
\hline U 089 & B & 23.03 .2006 & $10^{5}$ & $\mathrm{~F}$ & O6 \\
\hline U 090 & M & 25.03 .2006 & $10^{5}$ & M & $\mathrm{O} 2$ \\
\hline U 091 & $\mathrm{~B}$ & 27.03.2006 & $10^{6}$ & M & O-rough \\
\hline U 092 & $\mathrm{~K}$ & 29.03.2006 & $10^{6}$ & $\mathrm{~F}$ & O15 \\
\hline U 093 & $\mathrm{D}$ & 31.03 .2006 & $10^{6}$ & F & $\mathrm{O} 1$ \\
\hline U 094 & $\mathrm{E}$ & 04.04 .2006 & $10^{6}$ & F & PP-pool \\
\hline U 095 & $\mathrm{E}$ & 18.04.2006 & $10^{6}$ & $\mathrm{~F}$ & NT \\
\hline U 096 & $\mathrm{E}$ & 19.04 .2006 & $10^{6}$ & F & KK-pool \\
\hline U 097 & $\mathrm{E}$ & 19.04 .2006 & $10^{5}$ & $\mathrm{M} / \mathrm{CH}$ & O6 \\
\hline U 098 & $\mathrm{D}$ & 05.04 .2006 & $10^{5}$ & $\mathrm{~F}$ & O6 \\
\hline U 099 & $\mathrm{D}$ & 06.04 .2006 & $10^{6}$ & $\mathrm{~F}$ & O-rough \\
\hline U 100 & $\mathrm{C}$ & 26.04 .2006 & $10^{6}$ & F & MM-pool \\
\hline U 101 & $\mathrm{C}$ & 26.04 .2006 & $10^{4}$ & $\mathrm{~F}$ & $\mathrm{O} 75$ \\
\hline U 102 & $\mathrm{E}$ & 28.04.2006 & $10^{6}$ & $\mathrm{~F} / \mathrm{CH}$ & NT \\
\hline U 103 & $\mathrm{E}$ & 28.04.2006 & $10^{5}$ & $\mathrm{~F} / \mathrm{CH}$ & $\mathrm{O} 17$ \\
\hline U 104 & $\mathrm{E}$ & 28.04.2006 & $10^{6}$ & $\mathrm{~F}$ & NT \\
\hline U 105 & $\mathrm{E}$ & 28.04 .2006 & $10^{5}$ & $\mathrm{~F}$ & O6 \\
\hline
\end{tabular}


Table 2 (continued)

\begin{tabular}{llllll}
\hline Key & Department & Date [D-M-Y] & Bacteriuria & Sex & Serotype/O-group \\
\hline U 106 & E & 12.05 .2006 & $10^{6}$ & F & SS-pool \\
U 107 & H & 17.05 .2006 & $10^{6}$ & F & O-rough \\
U 108 & C & 17.05 .2006 & $10^{6}$ & F & O1 \\
U 109 & C & 05.09 .2006 & $10^{6}$ & F & O-rough \\
U 110 & C & 18.05 .2006 & $10^{6}$ & F & O2 \\
U 111 & E & 18.05 .2006 & $10^{6}$ & F & O-rough \\
U 112 & C & 21.04 .2006 & $10^{5}$ & F & O6 \\
U 113 & E & 10.05 .2006 & $10^{5}$ & F/CH & NT \\
U 114 & ND & ND & ND & ND & O6 \\
U 115 & ND & ND & ND & ND & O-rough \\
U 116 & E & 01.08 .2006 & $10^{6}$ & F/CH & NT \\
U 117 & L & 17.07 .2006 & $10^{6}$ & K & O1 \\
U 118 & K & 02.08 .2006 & $10^{6}$ & K & HH-pool \\
U 119 & F & 26.07 .2006 & $10^{6}$ & K & O8 \\
U 120 & C & 25.07 .2006 & $10^{6}$ & K & KK-pool \\
U 121 & C & 24.07 .2006 & $10^{6}$ & K & O78 \\
U 124 & C & C3.07.2006 & $10^{6}$ & K & NT \\
U 125 & C & 14.07 .2006 & $10^{6}$ & K & O-rough \\
U 126 & ND & ND & ND & ND & O-rough \\
U 127 & C & 13.07 .2006 & $10^{6}$ & K & OO-pool \\
U 128 & C & 06.09 .2006 & $10^{6}$ & K & GG-pool \\
U 130 & ND & ND & ND & ND & O-rough \\
\hline
\end{tabular}

Department: $A$ Clinic of cardiology and hypertension, $B$ Urology clinic, $C$ Urology office, $D$ Clinic of internal medicine, $E$ Primary medical care, $F$ Intensive care unit, $G$ Clinic of interventional cardiology, cardiac diabetology and cardiac rehabilitation, $H$ Clinic of neurology, $I$ Department of laboratory diagnostics, $J$ Clinic of neurosurgery, $K$ Trauma clinic, $L$ General surgery office, $M$ Emergency unit, $N$ Paid examination, private order

Sex: $F$ Female, $M$ Male, $C H$ Child, $N D$ No data obtained, $N T$ Non typable

O-pool detects the following serogroups: FF-pool O10, O11, O41, O100, O110, O148, O154, O159, O168, O179, GG-pool O49, O51, O52, O58, O61, O160, O176, O178, HH-pool O13, O17, O44, O62, O66, O73, O77, O106, KK-pool O24, O27, O29, O36, O56, O81, O169, O186, MM-pool O28ab, O28ac, O42, O112ab, O112ac, O142, O136, O144, OO-pool O34, O46, O85, O130, O134, O140, O167, O171, PP-pool O37, O39, O48, O59, O88, O91, O155, O165, O184, SS-pool O82, O87, O96, O105, O120, O137, O170, O183, UU-pool O86, O111, O114, O119, O125ab, O125ac, WW-pool O97, O98, O99, O104, O132, O139, $\mathrm{O} 147$

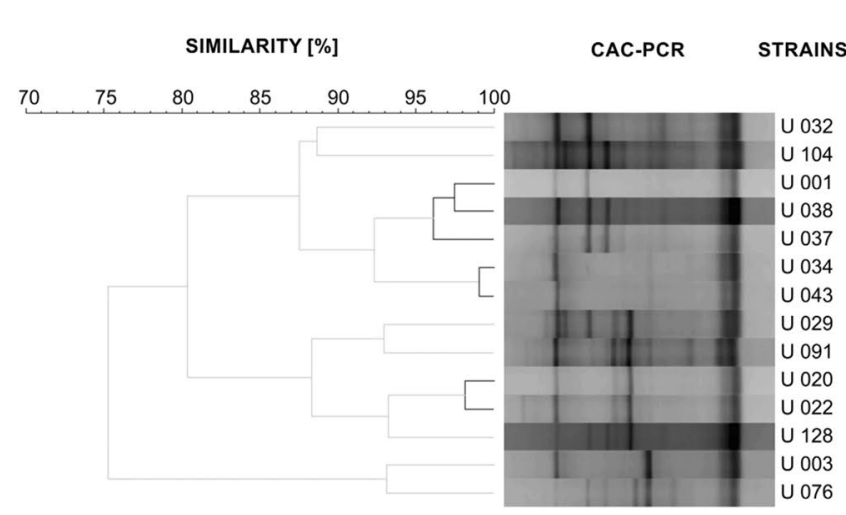

Fig. 1 Scheme depicting the assignment of numeric classes based on TRS-PCR profiles (here for CAC-PCR). The reproducibility for CAC-PCR exhibited 95,0\% (see Table 1S.), fingerprint patterns with

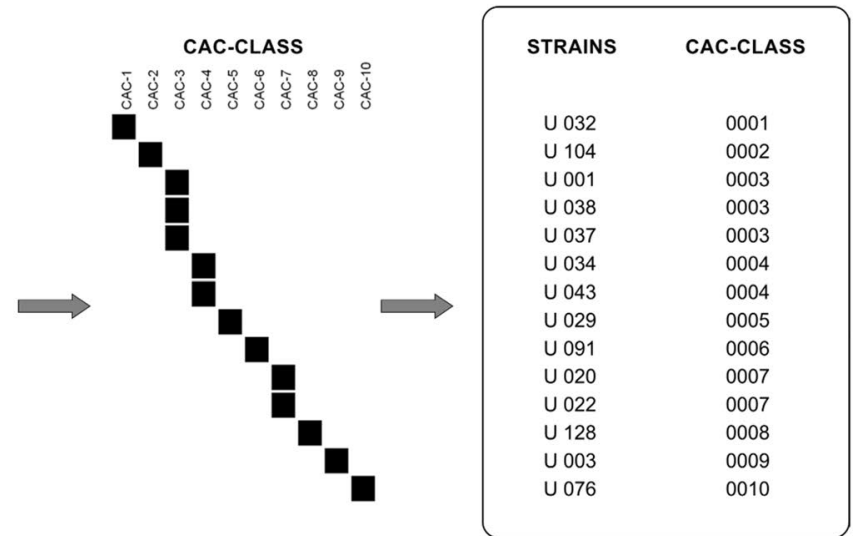

similarity above this value, were treated as identical. Based on analyses using BioNumerics software, each unique profile was assigned a class number-for these 14 strains $10 \mathrm{CAC}$ classes was assigned 
coli sub-typing based on the multiple TRS-PCR profiling, allowed the separation of 121 unique isolates from a collection of 124 strains. In this approach, we obtained numerical results which were easy to sort and interpret, allowing for easier analysis of these strains. Each test based on another TRS primer can bring something new to the analysis, but comparing the growing number of similarity dendrograms, it becomes difficult to interpret. Additionally, averaging them does not show detailed differences. In our new approach, we can test the effectiveness of several TRS markers at the same time, without the need to average the analyses from all TRS-PCR tests.

By analyzing the contents of the Table 1, it can be seen that strains U 058 and $\mathrm{U} 112$ are considered to be identical in TRS-PCR profiles despite the lack of one pathogenicity factor-papC (strain U 058). It can be inferred that these strains have lost one of their adhesines, without disturbing the structure of genome in the area we were investigating $[1,2,8]$. However, it cannot be ruled out that differentiation with an additional TRS marker would separate those strains. A more interesting case we observed for strains $\mathrm{U} 020$ and $\mathrm{U}$ 022, which have identical TRS-PCR profiles, despite differences in virulence composition. Strain U 020 possess astA, iutA and iroN, whereas stran $\mathrm{U} 022$ possess fyuA and iutA (Table 1). Apparently, there are no sequences in the genomic region where these genes are encoded, allowing for the generation of three specific types of TRS fragments.

In the group of tested strains, there were also isolates obtained from the same patient. Strains U 013 and U 046 isolated at different times had the same pathogenicity but differed within the CGG class. Such a small difference does not exclude the possibility of a re-infection with the same strain. The difference in one class may suggest a slight genetic change in the same strain, perhaps under the influence of antibiotic therapy, due to infection with a very similar strain or insufficient sensitivity of the method [1, 2, 8]. Among strains U 032, U 039 and U 097, also isolated from the same patient at different time points, the isolate $\mathrm{U} 032$ differs from the other two strains. In case of $U 039$ and $U$ 097 strains, there is a similar situation as for $\mathrm{U} 013$ and $\mathrm{U}$ 046 isolates. For such strains (U 013, U 046 and U 039, U 097), we recommend further subtyping, perhaps with an additional TRS marker.

Similarity dendrograms based on composite analysis of many tests usually show better resolution, but their interpretation may increase difficulties. We think that the proposed strategy in rendering TRS-PCR data, facilitates the interpretation of the obtained outcome. In addition, it could be done rapidly and results may be easier to compare, even between laboratories. It should be emphasized, with the method we used, we are able to demonstrate even small genetic differences among bacterial strains. Because the results are promising, the next stage of the research should include validation of the method: repeatability among laboratories working on different equipment and reagents.

In our research, we have repeatedly demonstrated the usefulness of the TRS-PCR profiling in bacterial genotyping [15-19]. However, strain differentiation, based on comparing single TRS-PCR results, or tracing differences resulting from averaged affinity trees (based on several TRS-PCR reactions), could also lead to evolutionary conclusions in addition to epidemiological investigations. To be sure, the results should be confronted with the results obtained from MLST analyses. Interestingly, we have shown in our recent studies that based on the analysis of TRS-PCR profiles, it is possible to predict directions of pathogenicity development in the E. coli population [25].

Funding These studies were partially funded by IMB PAS as part of the statutory research.

\section{Compliance with ethical standards}

Conflict of interest The authors declare that they have no conflict of interest.

Research involving human and animal participants This article does not contain any studies with human participants or animals performed by any of the authors.

Open Access This article is distributed under the terms of the Creative Commons Attribution 4.0 International License (http://creativeco mmons.org/licenses/by/4.0/), which permits unrestricted use, distribution, and reproduction in any medium, provided you give appropriate credit to the original author(s) and the source, provide a link to the Creative Commons license, and indicate if changes were made.

\section{References}

1. Pitout JD (2012) Extraintestinal pathogenic Escherichia coli: a combination of virulence with antibiotic resistance. Front Microbiol 3:9

2. Kaper JB, Nataro JP, Mobley HL (2004) Pathogenic Escherichia coli. Nat Rev Microbiol 2:123-140

3. Allocati N, Masulli M, Alexeyev MF, Di Ilio C (2013) Escherichia coli in Europe: an overview. Int J Environ Res Public Health 10(12):6235-6254

4. Johnson JR, Stell AL (2000) Extended virulence genotypes of Escherichia coli strains from patients with urosepsis in relation to phylogeny and host compromise. J Infect Dis 181:261-272. https ://doi.org/10.1086/315217

5. Bélanger L, Garenaux A, Harel J, Boulianne M, Nadeau E, Dozois CM (2011) Escherichia coli from animal reservoirs as a potential source of human extraintestinal pathogenic E. coli. FEMS Immunol Med Microbiol 62:1-10. https://doi.org/10.1111/j.1574695X.2011.00797.x

6. Johnson JR, Russo TA (2002) Extraintestinal pathogenic Escherichia coli: the other bad E coli. J Lab Clin Med 139:155-162

7. Foxman B (2010) The epidemiology of urinary tract infection. Nat Rev Urol 7:653-660. https://doi.org/10.1038/nrurol.2010.190 
8. Köhler CD, Dobrindt U (2011) What defines extraintestinal pathogenic Escherichia coli? Int J Med Microbiol 301:642-647. https ://doi.org/10.1016/j.ijmm.2011.09.006

9. Gündoğdu A, Long YB, Katouli M (2012) Prevalence and pathogenesis of extended-spectrum beta-lactamase producing Escherichia coli causing urinary tract infection in hospitalized patients. Eur J Clin Microbiol Infect Dis 31:3107-3116. https:// doi.org/10.1007/s10096-012-1672-0

10. Foxman B (2007) Contributions of molecular epidemiology to the understanding of infectious disease transmission, pathogenesis, and evolution. Ann Epidemiol 17:148-156. https://doi. org/10.1016/j.annepidem.2006.09.004

11. Russo TA, Johnson JR (2003) Medical and economic impact of extraintestinal infections due to Escherichia coli: focus on an increasingly important endemic problem. Microbes Infect 5:449-456

12. Johnson JR, Russo TA (2005) Molecular epidemiology of extraintestinal pathogenic (uropathogenic) Escherichia coli. Int J Med Microbiol 295:383-404. https://doi.org/10.1016/j. ijmm.2005.07.005

13. Bricker BJ (2011) Past, present and future of molecular technology applications for the epidemiology of bacterial diseases. J Anal Bioanal Tech. https://doi.org/10.4172/2155-9872.S10-001

14. Manges AR, Tellis PA, Vincent C, Lifeso K, Geneau G, ReidSmith RJ, Boerlin P (2009) Multi-locus variable number tandem repeat analysis for Escherichia coli causing extraintestinal infections. J Microbiol Methods 79:211-213. https://doi.org/10.1016/j. mimet.2009.09.006

15. Adamus-Bialek W, Wojtasik A, Majchrzak M, Sosnowski M, Parniewski P (2009) (CGG)4-based PCR as a novel tool for discrimination of uropathogenic Escherichia coli strains: comparison with enterobacterial repetitive intergenic consensus-PCR. J Clin Microbiol 47:3937-3944. https://doi.org/10.1128/JCM.01036-09

16. Kubiak-Szeligowska AB, Bartnicka M, Jarych D, Majchrzak M (2016) TRS-PCR profiling for discrimination of Escherichia coli strains isolated from children with diarrhea under 5 years of age in Lodz region. Poland Mol Biol Rep 43:871-880. https://doi. org/10.1007/s11033-016-4031-x

17. Majchrzak M, Krzyzanowska A, Kubiak AB, Wojtasik A, Wolkowicz T, Szych J, Parniewski P (2014) TRS-based PCR as a potential tool for inter-serovar discrimination of Salmonella Enteritidis, S. Typhimurium, S. Infantis, S. Virchow, S. Hadar, S. Newport and S. Anatum. Mol Biol Rep 41:7121-7132. https:// doi.org/10.1007/s11033-014-3592-9

18. Wojtasik A, Kubiak AB, Krzyzanowska A, Majchrzak M, Augustynowicz-Kopec E, Parniewski P (2012) Comparison of the (CCG)4-based PCR and MIRU-VNTR for molecular typing of Mycobacterium avium strains. Mol Biol Rep 39:7681-7686. https://doi.org/10.1007/s11033-012-1603-2

19. Wojtasik A, Majchrzak M, Adamus-Bialek W, AugustynowiczKopec E, Zwolska Z, Dziadek J, Parniewski P (2011) Trinucleotide repeat sequence-based PCR as a potential approach for genotyping Mycobacterium gordonae strains. J Microbiol Methods 85:28-32. https://doi.org/10.1016/j.mimet.2011.01.001

20. Clermont O, Christenson JK, Denamur E, Gordon DM (2013) The clermont Escherichia coli phylo-typing method revisited: improvement of specificity and detection of new phylo-groups. Environ Microbiol Rep 5:58-65. https://doi.org/10.1111/1758-2229.12019

21. Muller D, Greune L, Heusipp G, Karch H, Fruth A, Tschape H, Schmidt MA (2007) Identification of unconventional intestinal pathogenic Escherichia coli isolates expressing intermediate virulence factor profiles by using a novel single-step multiplex PCR. Appl Environ Microbiol 73:3380-3390. https://doi.org/10.1128/ AEM.02855-06

22. Bonacorsi S, Clermont O, Houdouin V, Cordevant C, Brahimi N, Marecat A, Tinsley C, Nassif X, Lange M, Bingen E (2003) Molecular analysis and experimental virulence of French and North American Escherichia coli neonatal meningitis isolates: identification of a new virulent clone. J Infect Dis 187(12):1895-1906

23. Restieri C, Garriss G, Locas MC, Dozois CM (2007) Autotransporter-encoding sequences are phylogenetically distributed among Escherichia coli clinical isolates and reference strains. Appl Environ Microbiol 73:1553-1562. https://doi.org/10.1128/AEM.01542 $-06$

24. Moulin-Schouleur M, Schouler C, Tailliez P, Kao MR, Bree A, Germon P, Oswald E, Mainil J, Blanco M, Blanco J (2006) Common virulence factors and genetic relationships between O18:K1:H7 Escherichia coli isolates of human and avian origin. J Clin Microbiol 44:3484-3492. https://doi.org/10.1128/ JCM.00548-06

25. Bartoszek K, Majchrzak M, Sakowski S, Kubiak-Szeligowska AB, Kaj I, Parniewski P (2018) Predicting pathogenicity behavior in Escherichia coli population through a state dependent model and TRS profiling. PLoS Comput Biol 14(1):e1005931

Publisher's Note Springer Nature remains neutral with regard to jurisdictional claims in published maps and institutional affiliations. 